\title{
Evaluación de la fertilidad de suelos en la granja escuela Casiciaco Haren Alde
}

\section{Ámbar del Pilar Medina- Moreno, Arturo Ariel Almanza- González² .}

${ }^{1}$ Investigadora principal, Facultad de Ingeniería y Tecnología, Universidad Católica Santa María La Antigua (USMA), Panamá, República de Panamá.

${ }^{2}$ Co investigador, Facultad de Ingeniería y Tecnología, Universidad Católica Santa María La Antigua (USMA), Panamá, República de Panamá.

*Autor para correspondencia. Email: 05ambar@hotmail.es

Recibido: 19 de julio de 2018

Aceptado: 10 de octubre de 2018

\begin{abstract}
The purpose of this study is to know the fertility of the different lands of the farm, by means of the sampling of soils and pits, in different areas, destined to paddocks, crops and soils at rest, which gave an idea of the level of deficiency or sufficiency of the major and minor elements of the floors. To do this, they chose two areas of pastures, two for agricultural use and two trees (coffee and forest), with different pioneer species, shrubs and trees, in a state of secondary ecological succession. For each zone sampling was carried out and pits were made in the areas of pasture with cattle and in the coffee area. No comparisons were made between the different sections; only comparisons were made within each area. We found deficiencies in major elements, and some minor ones, with an acidic $\mathrm{pH}$, product of the natural conditions of the place, and the sowing of crops, cattle raising and construction of infrastructures; these activities have caused erosion and compaction of the land. Currently, there was no study of soils on the farm, so it was necessary to start this research that will indicate the state of soils related to fertility; this will serve for the integral planning of the farm.
\end{abstract}

Keywords: edaphology, soil science, soil fertility, soil quality, tropical agriculture, Panamá.

\section{Resumen}

Este estudio tiene como propósito conocer la fertilidad de los diversos terrenos de la finca Casiciaco Haren Alde, mediante el muestreo de suelos y calicatas, en distintas áreas, destinadas a potreros, cultivos y suelos en descanso, lo que dio una idea del nivel de deficiencia o suficiencia de los elementos mayores y menores de los suelos. Para ello, escogieron dos zonas de potreros, dos de uso agrícola y dos arboladas (café y bosque), con diferentes especies pioneras, de arbustos y árboles, en estado de sucesión ecológica secundaria. Por cada zona se llevó a cabo la toma de muestra y se hizo calicatas en las zonas de potrero con bovino y en el área de café. No se hizo comparaciones entre las distintas secciones; solo se hicieron comparaciones dentro de cada área. Encontramos deficiencias en elementos mayores, y algunos menores, con un $\mathrm{pH}$ ácido, producto de las condiciones naturales del 
Invest. pens. crit. (ISSN 1812-3864)

Vol. 6, No. 3, septiembre- diciembre 2018

pp. $05-21$

lugar, y la siembra de cultivos, cría de ganado y construcción de infraestructuras; estas actividades han provocado erosión y compactación de los terrenos. Actualmente, no existía un estudio de suelos en la granja, por lo cual, fue necesario iniciar está investigación que indicará el estado de los suelos relacionados con la fertilidad; esto servirá para la planificación integral de la finca.

Palabras clave: edafología, ciencias del suelo, fertilidad de suelos, calidad de suelos, agricultura tropical, Panamá.

\section{Introducción}

La Granja Escuela Casiciaco Haren Alde (G.E.C.H.A), es el resultado del esfuerzo y del compromiso de varias instituciones nacionales e internacionales para proporcionar al productor, una alternativa productiva desde una óptica de sostenibilidad y desarrollo humano. El enfoque de producción que se ha aplicado es de agricultura orgánica, repelentes orgánicos, y ganadería sostenible, sin aplicación de abonos químicos. Se siembran granos, hortalizas, tubérculos y otros cultivos, además se cría ganado bovino y porcino; gallinas y otras especies menores. Estas actividades han incidido en la fertilidad de los suelos, con posibilidades de erosión y compactación en los terrenos. Actualmente, se requiere hacer investigaciones para determinar las condiciones de erosión y fertilidad de los suelos, con el objetivo de programar, más eficazmente, la aplicación de abonos; aumentar las cosechas; y evitar el deterioro a largo plazo, de los terrenos de siembra y cría de ganado bovino (A. González, Comunicación Personal, 24 de julio de 2018). Se distinguen tres grupos de nutrientes: Macronutrientes: son requeridos en cantidades sustanciales; micronutrientes: se necesitan en cantidades pequeñas; y elementos trazas: indispensables en cantidades mínimas (Grijpma, P; et al., 1982). Los suelos más comunes encontrados en la región de Azuero, se clasifican como Udic Dystropepts, umbric dystropepts, udic tropudalf, vertic hapludalf vertic dystrudepts. Todos originados de rocas ígneas, lo que explica el ¿por qué? de los bajos niveles de nutrimentos en muchos de estos (CATAPAN, 1970, citado por Villarreal, et al, $\mathrm{s} / \mathrm{f}$ ). En los suelos de áreas de clima tropical, el contenido en nitrógeno varía ampliamente entre 0.02\% y $0.04 \%$ (Fassbender\& Bornemisza, 1994, p. 199-200). El contenido total de fósforo en los suelos va de 0.03 a 0.30 por ciento (Chapman, 1991, p.108). De los trece elementos esenciales obtenidos del suelo por las plantas, seis son usadas relativamente por grandes cantidades, y, por consiguiente, merecen nuestra atención primordial. Son: nitrógeno, fósforo, potasio, calcio, magnesio y azufre. Se designan con el nombre de macronutrientes porque las plantas los utilizan en grandes cantidades. El crecimiento de las plantas puede ser retardado por varias causas: porque hay escasez de ellos en el suelo, porque resultan asimilables en forma muy lenta, o porque no estén adecuadamente equilibrados por los otros 22 elementos nutritivos. A veces pueden existir las tres limitaciones juntas. Esto es cierto, con frecuencia, respecto al nitrógeno. (Buckman \& Brady, 1996, p.21). Los siete micronutrimentos que acompañan a los nueve macronutrimentos; son esenciales para que las plantas realicen sus funciones fisiológicas y metabólicas. Ellos son: hierro, manganeso, cobre, cinc, boro, molibdeno y cloro. Todos son mejor absorbidos por las raíces de las plantas en condiciones de $\mathrm{pH}$ ácido; excepto el molibdeno, que, con ese valor de $\mathrm{pH}$, es fuertemente fijado por óxidos hidratados de hierro y aluminio. Por esa razón, el molibdeno está más disponible en el suelo, a pH neutro o alcalino. Los primeros cuatro elementos, cobre, hierro, manganeso y cinc, se conocen como elementos de transición (kass, 2007, p.19). El objetivo general fue determinar los niveles de fertilidad de los suelos, con el fin de indicar deficiencias y grados satisfactorios, en los nutrientes de los terrenos de la Granja Escuela Casiciaco Haren Alde. 
Invest. pens. crit. (ISSN 1812-3864)

Vol. 6, No. 3, septiembre- diciembre 2018

pp. $05-21$

\section{Metodología}

El estudio se llevó a cabo en los terrenos de la Granja Escuela Casiciaco Haren Alde ubicada en el Corregimiento de Las Minas, Distrito de Las Minas, Provincia de Herrera, República de Panamá.

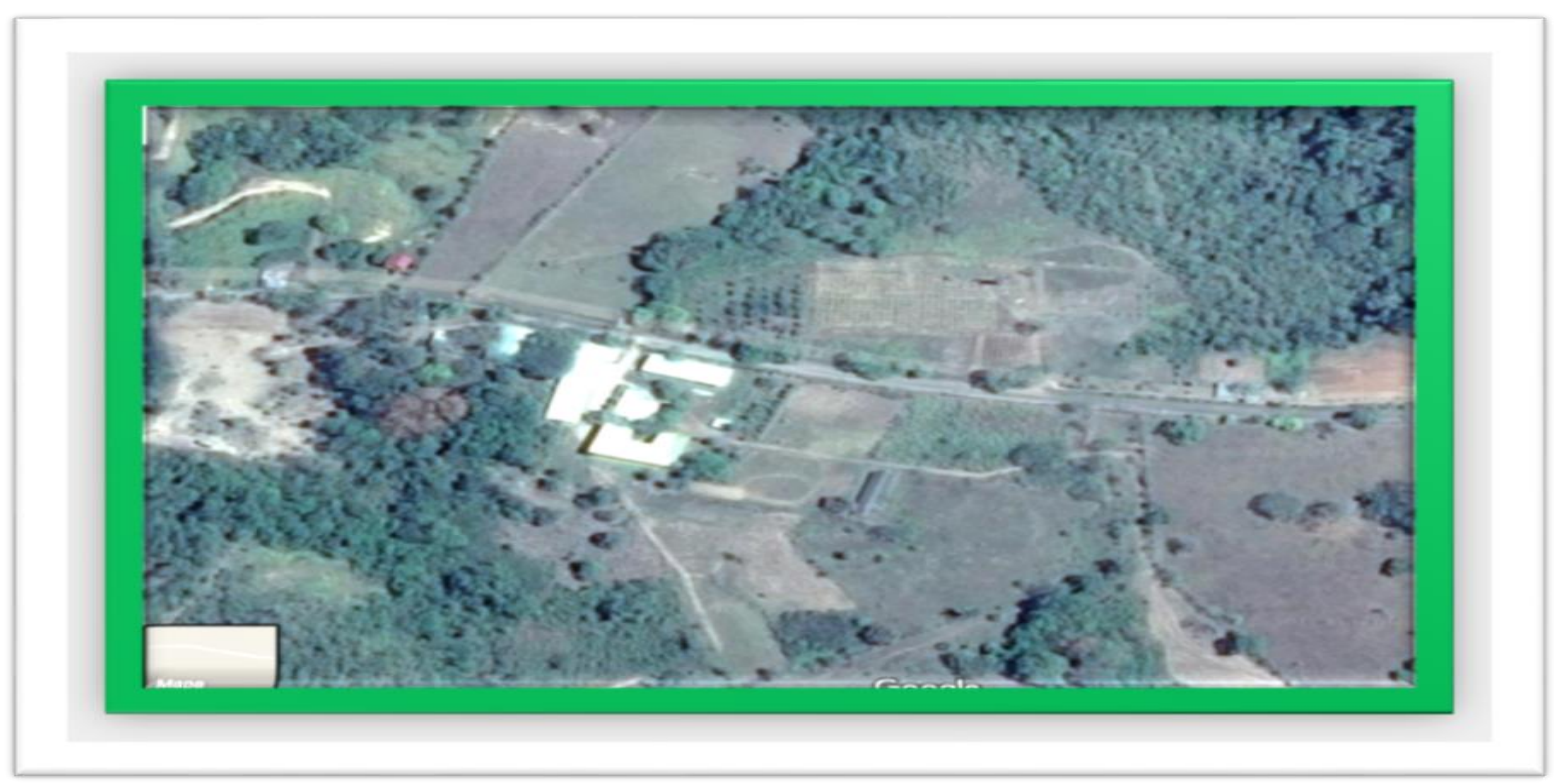

Figura 1 Vista aérea de la Granja Escuela Casiciaco Haren Alde.

Se seleccionaron 6 parcelas, que representaban diferentes usos de suelos, dentro de la finca, dedicados a cultivos de maíz, cultivo de plátano asociado con otoe, cultivo de café, potreros (en descanso y con bovinos) y área de bosque; a cada una se le tomó un punto en coordenadas UTM.

A cada una de las parcelas se les realizó un muestreo de suelo que consistió en 10 sub muestras, en zigzag. A lo largo de cada uno, se homogenizaron las mismas para obtener la muestra representativa, que fue llevada al laboratorio especializado de análisis de la Universidad Nacional de Panamá, ubicado en el Centro Regional Universitario de Los Santos. Los análisis trabajados en el laboratorio fueron por color, según el método de la tabla de colorimetría de Munsell, textura por el método Bouyoucos, $\mathrm{pH}$ mediante el método de solución de suelo en agua destilada (1:2.5), determinación de materia orgánica, de acuerdo el método de Walkley- Black, fósforo por el método carolina del norte y todo el resto de los elementos atendiendo el método de espectrofotometría de llamas.

Se trabajaron 2 calicatas, de $1 \mathrm{~m}$ de largo por $1 \mathrm{~m}$ de ancho, y $1 \mathrm{~m}$ de profundidad, en la parcela de café, y la otra en potrero, con bovinos. Una de las calicatas no alcanzó la profundidad de un metro. De estas calicatas se tomaron muestras de cada perfil y se llevaron para su análisis. Con la información, recolectada, se determinó la condición de fertilidad de los suelos de la granja. 
Invest. pens. crit. (ISSN 1812-3864)

Vol. 6, No. 3, septiembre- diciembre 2018

pp. $05-21$

\section{Resultados}

Cuadro 1. Usos de suelos, Tesis: Evaluación de la Fertilidad de Suelos en la Granja Escuela Casiciaco Haren Alde, distrito de Las Minas, provincia de Herrera, República de Panamá, 2018.

\begin{tabular}{|c|c|}
\hline Usos De Suelos & Áreas \\
\hline Cultivos & 2 \\
\hline Potrero & 2 \\
\hline Café & 1 \\
\hline Bosques & 1 \\
\hline
\end{tabular}

Para el desarrollo de la investigación se evaluaron los suelos de dos áreas de cultivos y de potrero; un área de café y de bosque; en una de las áreas de cultivo se había sembrado, anteriormente, maíz, y en el otro, plátano y otoe; en las áreas de potrero, uno estaba en "descanso", y el otro con bovino; el café tiene como sombra la caoba africana. El área boscosa, se compone de guácimo, guarumo, canillo, malagueta, y nance, se encuentra en sucesión ecológica secundaria hace 22 años (cuadro 1).

Cuadro 2. Coordenadas UTM, Tesis: Evaluación de la Fertilidad de Suelos en la Granja Escuela Casiciaco Haren Alde, distrito de las Minas, provincia de Herrera, República de Panamá, 2018.

\begin{tabular}{ll|l} 
Áreas & \multicolumn{2}{c}{ Coordenadas UTM } \\
& Latitud & Longitud \\
Cultivo de Maíz & 526723 & 862421 \\
Cultivo de Plátano y Otoe & 526717 & 862414 \\
Potrero en “descanso" & 526372 & 862853 \\
Potrero con Bovino & 526775 & 862350 \\
Café & 526625 & 862427 \\
Bosques & 526832 & 862529
\end{tabular}

Se presentan los lugares de estudio, representadas en coordenadas UTM (cuadro 2).

Cuadro 3. Parámetros de Análisis de Suelos, Cultivo de Maíz, Tesis: Evaluación de la Fertilidad de Suelos en la Granja Escuela Casiciaco Haren Alde, distrito de las Minas, provincia de Herrera, República de Panamá, 2018.

\begin{tabular}{|c|c|c|c|}
\hline \multirow{2}{*}{ Parámetros } & \multicolumn{2}{|c|}{ Resultado } & \multirow{2}{*}{ Interpretación } \\
\cline { 2 - 3 } & $\mathrm{ppm}$ & $\mathrm{meq} / 100 \mathrm{~g}$ & \\
\hline Fósforo $(\mathrm{P})$ & 0,00 & & TRAZA \\
\hline Potasio $(\mathrm{K})$ & 82,41 & 0,21 & MEDIO \\
\hline Calcio $(\mathrm{Ca})$ & 82,74 & 0,41 & BAJO \\
\hline
\end{tabular}


Invest. pens. crit. (ISSN 1812-3864)

Vol. 6, No. 3, septiembre- diciembre 2018

pp. 05-21

\begin{tabular}{|c|c|c|c|}
\hline Magnesio $(\mathrm{Mg})$ & 45,54 & 0,38 & BAJO \\
\hline Sodio $(\mathrm{Na})$ & 0,00 & 0,00 & MEDIO \\
\hline Hierro $(\mathrm{Fe})$ & 60,34 & & MEDIO \\
\hline Manganeso $(\mathrm{Mn})$ & 47,57 & & BAJO \\
\hline Cobre $(\mathrm{Cu})$ & 0,00 & & BAJO \\
\hline Zinc $(\mathrm{Zn})$ & 2,45 & & BAJO \\
\hline Aluminio $(\mathrm{Al})$ & & 0,10 & BAJO \\
\hline Acidez & & 0,20 & \multicolumn{1}{|c|}{} \\
\hline CICE & & 1,10 & CIC \\
\hline
\end{tabular}

Observaciones: $\mathrm{Ppm}=$ parte por millón; $\mathrm{meq}=$ mili equivalente; $\mathrm{CICE}=$ capacidad de intercambio catiónica efectiva.

En relación a los macroelementos, los suelos del cultivo de maíz presentaron niveles bajos de magnesio y calcio, medio de potasio y trazas de fósforos, indicando baja fertilidad; en referencia a los microelementos, se obtuvieron niveles bajos de aluminio, zinc y cobre, y medio de manganeso y hierro, señalando una influencia del hierro sobre la acidez del suelo; la capacidad de intercambio catiónico efectiva presentó un nivel bajo, lo que indica un grado bajo de bases intercambiables (Cuadro 3).

Cuadro 4. Saturación de Bases en el Análisis de Suelo, Cultivo de Maíz, Tesis: Evaluación de la Fertilidad de Suelos en la Granja Escuela Casiciaco Haren Alde, distrito de las Minas, provincia de Herrera, República de Panamá, 2018.

\begin{tabular}{|c|c|}
\hline \multicolumn{2}{|c|}{ Saturación de bases \% } \\
\hline Potasio $(\mathrm{k})$ & 19,12 \\
\hline Calcio $(\mathrm{Ca})$ & 37,53 \\
\hline Magnesio $(\mathrm{Mg})$ & 34,29 \\
\hline Sodio $(\mathrm{Na})$ & 0,00 \\
\hline Acidez & 9,07 \\
\hline \multicolumn{2}{|c|}{} \\
\hline $\mathrm{Ca} / \mathrm{Mg}$ & 1,09 \\
\hline $\mathrm{Mg} / \mathrm{k}$ & 1,79 \\
\hline $\mathrm{Ca}+\mathrm{Mg} / \mathrm{k}$ & 3,76 \\
\hline $\mathrm{Ca} / \mathrm{k}$ & 1,96 \\
\hline
\end{tabular}


Invest. pens. crit. (ISSN 1812-3864)

Vol. 6, No. 3, septiembre- diciembre 2018

pp. $05-21$

En relación a los cationes principales (potasio, calcio, magnesio, sodio), en el suelo del cultivo de maíz, se muestra un nivel bajo de calcio, un nivel alto de potasio y magnesio, y un nivel aceptable de sodio. En el criterio de saturación de base, nos indica que este suelo tiene $\mathrm{pH}$ muy ácido, debido a que la suma de sus bases fue menor a 50\%. Además, demuestra un desbalance en la relación del calcio, respecto al magnesio, y en un nivel adecuado para el potasio en la relación $\mathrm{Ca}+\mathrm{Mg} / \mathrm{k}$ (Cuadro 4).

Cuadro 5. Parámetros de Análisis de Suelos, Cultivo de Plátano y Otoe, Tesis: Evaluación de la Fertilidad de Suelos en la Granja Escuela Casiciaco Haren Alde, distrito de las Minas, provincia de Herrera, República de Panamá, 2018.

\begin{tabular}{|c|c|c|c|}
\hline \multirow{2}{*}{ Parámetros } & \multicolumn{2}{|c|}{ Resultado } & \multirow{2}{*}{ Interpretación } \\
\hline & ppm & meq/100g & \\
\hline Fósforo $(\mathrm{P})$ & 0,00 & & TRAZA \\
\hline $\operatorname{Potasio}(\mathrm{K})$ & 111,14 & 0,28 & MEDIO \\
\hline Calcio (Ca) & 81,80 & 0,41 & BAJO \\
\hline Magnesio (Mg) & 54,32 & 0,45 & BAJO \\
\hline Sodio $(\mathrm{Na})$ & 0,00 & 0,00 & \\
\hline Hierro $(\mathrm{Fe})$ & 39,61 & & MEDIO \\
\hline Manganeso (Mn) & 55,70 & & ALTO \\
\hline Cobre $(\mathrm{Cu})$ & 0,08 & & BAJO \\
\hline Zinc $(\mathrm{Zn})$ & 5,00 & & MEDIO \\
\hline Aluminio (Al) & & 0,10 & BAJO \\
\hline Acidez & & 0,20 & BAJO \\
\hline CICE & & 1,14 & \\
\hline
\end{tabular}

En correlación, a los macroelementos, los suelos del cultivo de Plátano y Otoe, mostraron niveles bajos de magnesio y calcio, medio de potasio, y trazas de fósforos, indicando baja fertilidad. En los que corresponden a los microelementos, se obtuvieron niveles bajos de aluminio y cobre, medio de zinc, hierro, y un manganeso con nivel alto; indicando una influencia del hierro sobre la acidez del suelo; la capacidad de intercambio catiónico efectiva presentó un nivel bajo, lo que indica un grado bajo de bases intercambiables (Cuadro 5).

Cuadro 6. Saturación de Bases en el Análisis de Suelo, Cultivo de Plátano y Otoe, Tesis: Evaluación de la Fertilidad de Suelos en la Granja Escuela Casiciaco Haren Alde, distrito de las Minas, provincia de Herrera, República de Panamá. 2018 
Invest. pens. crit. (ISSN 1812-3864)

Vol. 6, No. 3, septiembre- diciembre 2018

pp. 05-21

\begin{tabular}{|c|c|}
\hline \multicolumn{2}{|c|}{ Saturación de bases $\%$} \\
\hline Potasio $(\mathrm{k})$ & 24,84 \\
\hline Calcio $(\mathrm{Ca})$ & 35,75 \\
\hline Magnesio $(\mathrm{Mg})$ & 39,41 \\
\hline Sodio $(\mathrm{Na})$ & 0,00 \\
\hline Acidez & 0,00 \\
\hline \multicolumn{2}{|c|}{} \\
\hline $\mathrm{Ca} / \mathrm{Mg}$ & 0,91 \\
\hline $\mathrm{Mg} / \mathrm{k}$ & 1,59 \\
\hline $\mathrm{Ca}+\mathrm{Mg} / \mathrm{k}$ & 3,03 \\
\hline $\mathrm{Ca} / \mathrm{k}$ & 1,44 \\
\hline
\end{tabular}

En referencia a las bases de saturación (potasio, calcio, magnesio, sodio), en el cultivo de Plátano y Otoe, se presentó un nivel bajo de calcio, un nivel alto de potasio y magnesio, un nivel aceptable de sodio. Al igual se notó una deficiencia de calcio con respecto al magnesio, ya que el Ca debe ser tres veces más que el valor del $\mathrm{Mg}$, en la relación $\mathrm{Ca}+\mathrm{Mg} / \mathrm{k}$, contiene un nivel aceptable para el potasio, las demás relaciones son adecuadas. Además, se denota un suelo con un $\mathrm{pH}$ muy ácido, según el criterio de bases. (Cuadro 6)

Cuadro 7. Parámetros de Análisis de Suelos, Café, Tesis: Evaluación de la Fertilidad de Suelos en la Granja Escuela Casiciaco Haren Alde, distrito de las Minas, provincia de Herrera, República de Panamá, 2018.

\begin{tabular}{|c|c|c|c|}
\hline \multirow{2}{*}{ Parámetros } & \multicolumn{2}{|c|}{ Resultado } & \multirow{2}{*}{ Interpretación } \\
\cline { 2 - 3 } & $\mathrm{ppm}$ & $\mathrm{meq} / 100 \mathrm{~g}$ & \\
\hline Fósforo $(\mathrm{P})$ & 0,00 & & TRAZA \\
\hline Potasio $(\mathrm{K})$ & 131,97 & 0,34 & MEDIO \\
\hline Calcio $(\mathrm{Ca})$ & 85,53 & 0,43 & BAJO \\
\hline Magnesio $(\mathrm{Mg})$ & 80,34 & 0,67 & MEDIO \\
\hline Sodio $(\mathrm{Na})$ & 0,00 & 0,00 & MEDIO \\
\hline Hierro $(\mathrm{Fe})$ & 40,53 & & MEDIO \\
\hline Manganeso $(\mathrm{Mn})$ & 19,79 & & BAJO \\
\hline Cobre $(\mathrm{Cu})$ & 0,00 & & \\
\hline
\end{tabular}


Invest. pens. crit. (ISSN 1812-3864)

Vol. 6, No. 3, septiembre- diciembre 2018

pp. $05-21$

\begin{tabular}{|c|c|c|c|}
\hline Zinc $(\mathrm{Zn})$ & 4,20 & & BAJO \\
\hline Aluminio (Al) & & 0,00 & BAJO \\
\hline Acidez & & 0,20 & BAJO \\
\hline CICE & & 1,43 & \multicolumn{2}{c}{} \\
\hline
\end{tabular}

Con respecto a los macroelementos, los suelos del café, indicó un nivel bajo de calcio, medio de potasio y magnesio, y trazas de fosforo, lo que demuestra, que la alta cantidad de lluvia que se dan en estas zonas, provoca un nivel bajo de Ca. En cuanto a los microelementos, se mostró niveles bajo de cobre, zinc, y aluminio, medio de hierro y manganeso, señalando baja fertilidad y una influencia del hierro sobre la acidez del suelo; la capacidad de intercambio catiónico efectiva presentó un nivel bajo, lo que indica un grado bajo de bases intercambiables. (Cuadro 7)

Cuadro 8. Saturación de Bases en el Análisis de Suelo, Café, Tesis: Evaluación de la Fertilidad de Suelos en la Granja Escuela Casiciaco Haren Alde, distrito de las Minas, provincia de Herrera, República de Panamá, 2018.

\begin{tabular}{|c|c|}
\hline \multicolumn{2}{|c|}{ Saturación de bases \% } \\
\hline Potasio (k) & 23,57 \\
\hline Calcio $(\mathrm{Ca})$ & 29,86 \\
\hline Magnesio $(\mathrm{Mg})$ & 46,57 \\
\hline Sodio $(\mathrm{Na})$ & 0,00 \\
\hline Acidez & 0,00 \\
\hline \multicolumn{2}{|c|}{ Relaciones } \\
\hline $\mathrm{Ca} / \mathrm{Mg}$ & \\
\hline $\mathrm{Mg} / \mathrm{k}$ & 0,64 \\
\hline $\mathrm{Ca}+\mathrm{Mg} / \mathrm{k}$ & 1,98 \\
\hline $\mathrm{Ca} / \mathrm{k}$ & 3,24 \\
\hline
\end{tabular}

En referencia a las bases de saturación (potasio, calcio, magnesio, sodio), en el cultivo de café, se presentó un nivel bajo de calcio, un nivel alto de potasio y magnesio, y un nivel aceptable de sodio. Se mostró una deficiencia de calcio en la relación $\mathrm{Ca} / \mathrm{Mg}$; en cuanto a la relación $\mathrm{Ca}+\mathrm{Mg} / \mathrm{k}$, indica un nivel aceptable para el potasio, las otras relaciones tienen un nivel aceptable. Además, según el criterio de saturación de bases, con resultado menor a 50\%, señala un pH muy ácido en el suelo (Cuadro 8).

Cuadro 9. Parámetros de Análisis de Suelos, Bosque, Tesis: Evaluación de la Fertilidad de Suelos en la Granja Escuela Casiciaco Haren Alde, distrito de las Minas, provincia de Herrera, República de Panamá, 2018. 
Invest. pens. crit. (ISSN 1812-3864)

Vol. 6, No. 3, septiembre- diciembre 2018

pp. 05-21

\begin{tabular}{|c|c|c|c|}
\hline \multirow{2}{*}{ Parámetros } & \multicolumn{2}{|c|}{ Resultado } & \multirow{2}{*}{ Interpretación } \\
\cline { 2 - 3 } & $\mathrm{ppm}$ & $\mathrm{meq} / 100 \mathrm{~g}$ & TRAZA \\
\hline Fósforo $(\mathrm{P})$ & 0,00 & & MEDIO \\
\hline Potasio(K) & 115,45 & 0,30 & BAJO \\
\hline Calcio $(\mathrm{Ca})$ & 32,01 & 0,16 & BAJO \\
\hline Magnesio $(\mathrm{Mg})$ & 31,90 & 0,26 & ALTO \\
\hline Sodio $(\mathrm{Na})$ & 0,00 & 0,00 & MEDIO \\
\hline Hierro $(\mathrm{Fe})$ & 91,11 & & MEDIO \\
\hline Manganeso $(\mathrm{Mn})$ & 35,45 & & BAJO \\
\hline Cobre $(\mathrm{Cu})$ & 5,98 & & MEDIO \\
\hline Zinc $(\mathrm{Zn})$ & 3,69 & 1,00 & BAJO \\
\hline Aluminio $(\mathrm{Al})$ & & 0,20 & \\
\hline Acidez & & 1,72 & \\
\hline CICE & & & \\
\hline
\end{tabular}

Considerando los macroelementos, los suelos del bosque, presentan bajos niveles de calcio y magnesio, medio de potasio y trazas de fósforos, indicando baja fertilidad; en relación a los microelementos, muestran niveles bajos de zinc, medio de manganeso, cobre y aluminio, y alto de hierro; señalando una influencia del hierro sobre la acidez del suelo; la capacidad de intercambio catiónico efectiva presentó un nivel bajo, lo que indica un grado bajo de bases intercambiables con un suelo muy pobre (Cuadro 9).

Cuadro 10. Saturación de Bases en el Análisis de Suelo, Bosque, Tesis: Evaluación de la Fertilidad de Suelos en la Granja Escuela Casiciaco Haren Alde, distrito de las Minas, provincia de Herrera, República de Panamá, 2018.

\begin{tabular}{|c|c|}
\hline \multicolumn{2}{|c|}{ Saturación de bases \% } \\
\hline Potasio $(\mathrm{k})$ & 17,17 \\
\hline Calcio $(\mathrm{Ca})$ & 9,30 \\
\hline Magnesio $(\mathrm{Mg})$ & 15,39 \\
\hline Sodio $(\mathrm{Na})$ & 0,00 \\
\hline Acidez & 58,14 \\
\hline \multicolumn{2}{|c|}{ Relaciones } \\
\hline $\mathrm{Ca} / \mathrm{Mg}$ & 0,60 \\
\hline $\mathrm{Mg} / \mathrm{k}$ & 0,90 \\
\hline
\end{tabular}


Invest. pens. crit. (ISSN 1812-3864)

Vol. 6, No. 3, septiembre- diciembre 2018

pp. $05-21$

\begin{tabular}{|c|c|}
\hline $\mathrm{Ca}+\mathrm{Mg} / \mathrm{k}$ & 1,44 \\
\hline $\mathrm{Ca} / \mathrm{k}$ & 0,54 \\
\hline
\end{tabular}

Los cationes principales (potasio, calcio, magnesio, sodio), en los suelos del bosque, mostraron un nivel alto de potasio, bajo de calcio y un grado aceptable de magnesio y sodio. En el criterio de saturación de base, nos indica que este suelo es muy ácido, debido a que la suma de estas bases es menor a $50 \%$. Igualmente, se notó una deficiencia de $\mathrm{Ca}$ en la relación $\mathrm{Ca} / \mathrm{Mg}$, y una deficiencia de magnesio en la relación $\mathrm{Mg} / \mathrm{k}$, en la relación $\mathrm{Ca}+\mathrm{Mg} / \mathrm{k}$, hay nivel adecuado para el potasio, las demás relaciones son aceptables (Cuadro 10).

Cuadro 11. Parámetros de Análisis de Suelos, Potrero en "descanso", Tesis: Evaluación de la Fertilidad de Suelos en la Granja Escuela Casiciaco Haren Alde, distrito de las Minas, provincia de Herrera, República de Panamá, 2018.

\begin{tabular}{|c|c|c|c|}
\hline \multirow{2}{*}{ Parámetros } & \multicolumn{2}{|c|}{ Resultado } & \multirow{2}{*}{ Interpretación } \\
\cline { 2 - 3 } & $\mathrm{ppm}$ & $\mathrm{meq} / 100 \mathrm{~g}$ & \multirow{2}{*}{ TRAZA } \\
\hline Fósforo $(\mathrm{P})$ & 0,00 & 0,30 & MEDIO \\
\hline Potasio $(\mathrm{K})$ & 116,64 & 0,32 & BAJO \\
\hline Calcio $(\mathrm{Ca})$ & 64,88 & 0,40 & BAJO \\
\hline Magnesio $(\mathrm{Mg})$ & 48,20 & 0,00 & \multirow{2}{*}{ ALTO } \\
\hline Sodio $(\mathrm{Na})$ & 0,00 & & ALTO \\
\hline Hierro $(\mathrm{Fe})$ & 102,54 & & ALTO \\
\hline Manganeso $(\mathrm{Mn})$ & 79,44 & & BAJO \\
\hline Cobre $(\mathrm{Cu})$ & 8,76 & 0,10 & BAJO \\
\hline Zinc $(\mathrm{Zn})$ & 3,91 & 0,20 & BAJO \\
\hline Aluminio $(\mathrm{Al})$ & & 1,12 & \multirow{2}{*}{} \\
\hline Acidez & & & \\
\hline CICE & & & \\
\hline
\end{tabular}

Tomando en cuenta los macroelementos, en los suelos del potrero en "descanso", se mostró niveles bajos de calcio y magnesio, medio de potasio, y trazas de fósforo, lo que nos indica una baja fertilidad en los terrenos. En cuanto a los microelementos, se presentaron niveles bajos de aluminio y zinc, un nivel alto de hierro, manganeso y cobre; indicando una influencia del hierro sobre la acidez del suelo; la capacidad de intercambio catiónico efectiva presentó un nivel bajo, lo que indica un grado bajo de bases intercambiables con un suelo muy pobre (Cuadro 11). 
Invest. pens. crit. (ISSN 1812-3864)

Vol. 6, No. 3, septiembre- diciembre 2018

pp. $05-21$

Cuadro 12. Saturación de Bases en el Análisis de Suelo, Potrero en "descanso", Tesis: Evaluación de la Fertilidad de Suelos en la Granja Escuela Casiciaco Haren Alde, distrito de las Minas, provincia de Herrera, República de Panamá, 2018.

\begin{tabular}{|c|c|}
\hline \multicolumn{2}{|c|}{ Saturación de bases $\%$} \\
\hline Potasio (k) & 26,57 \\
\hline Calcio (Ca) & 28,89 \\
\hline Magnesio $(\mathrm{Mg})$ & 35,63 \\
\hline Sodio $(\mathrm{Na})$ & 0,00 \\
\hline Acidez & 8,91 \\
\hline \multicolumn{2}{|c|}{ Relaciones } \\
\hline $\mathrm{Ca} / \mathrm{Mg}$ & 0,81 \\
\hline $\mathrm{Mg} / \mathrm{k}$ & 1,34 \\
\hline $\mathrm{Ca}+\mathrm{Mg} / \mathrm{k}$ & 2,43 \\
\hline $\mathrm{Ca} / \mathrm{k}$ & 1,09 \\
\hline
\end{tabular}

Las bases de saturación (potasio, calcio, magnesio, sodio), en los suelos del potrero en "descanso", revelan un alto nivel de potasio y magnesio, nivel bajo de calcio y un nivel aceptable de sodio. Se denota según el criterio de saturación de bases, un resultado menor a $50 \%$, indicando un $\mathrm{pH}$ neutro o básico; además, indica una carencia de Ca en la relación $\mathrm{Ca} / \mathrm{Mg}$, muestra un nivel, adecuado, para el potasio, en la relación $\mathrm{Ca}+\mathrm{Mg} / \mathrm{k}$, en cuanto las demás relaciones se consideran aceptables (Cuadro 12).

Cuadro 13. Parámetros de Análisis de Suelos, Potrero con Bovino, Tesis: Evaluación de la Fertilidad de Suelos en la Granja Escuela Casiciaco Haren Alde, distrito de las Minas, provincia de Herrera, República de Panamá, 2018.

\begin{tabular}{|c|c|c|c|}
\hline \multirow{2}{*}{ Parámetros } & \multicolumn{2}{|c|}{ Resultado } & \multirow{2}{*}{ Interpretación } \\
\cline { 2 - 3 } & $\mathrm{ppm}$ & $\mathrm{meq} / 100 \mathrm{~g}$ & \\
\hline Fósforo $(\mathrm{P})$ & 0,00 & & TRAZA \\
\hline Potasio $(\mathrm{K})$ & 48,07 & 0,12 & BAJO \\
\hline Calcio $(\mathrm{Ca})$ & 73,19 & 0,37 & BAJO \\
\hline Magnesio $(\mathrm{Mg})$ & 45,99 & 0,38 & BAJO \\
\hline Sodio $(\mathrm{Na})$ & 0,00 & 0,00 & ALTO \\
\hline Hierro $(\mathrm{Fe})$ & 136,52 & & ALTO \\
\hline Manganeso $(\mathrm{Mn})$ & 125,19 & & \\
\hline
\end{tabular}


Invest. pens. crit. (ISSN 1812-3864)

Vol. 6, No. 3, septiembre- diciembre 2018

pp. $05-21$

\begin{tabular}{|c|c|c|c|}
\hline Cobre $(\mathrm{Cu})$ & 10,29 & & ALTO \\
\hline Zinc $(\mathrm{Zn})$ & 10,31 & & MEDIO \\
\hline Aluminio (Al) & & 0,20 & BAJO \\
\hline Acidez & & 0,20 & BAJO \\
\hline CICE & & 1,07 & \multicolumn{2}{|c}{} \\
\hline
\end{tabular}

Referente a los macroelementos, en los suelos de potrero con bovino, se manifiestan niveles bajo de potasio, calcio, magnesio y trazas de fósforo, lo que nos muestra una baja fertilidad. Considerando los microelementos, indicaron un nivel bajo de aluminio, medio de zinc y altos niveles de hierro, cobre y magnesio. Dando a demostrar la influencia que conlleva el hierro en la acidez de esta parcela. La capacidad de intercambio catiónico efectiva mostró un nivel bajo, lo que indica un grado bajo de bases intercambiables con un suelo muy pobre (Cuadro 13).

Cuadro 14. Saturación de Bases en el Análisis de Suelo, Potrero con bovino, Tesis: Evaluación de la Fertilidad de Suelos en la Granja Escuela Casiciaco Haren Alde, distrito de las Minas, provincia de Herrera, República de Panamá, 2018.

\begin{tabular}{|c|c|}
\hline \multicolumn{2}{|c|}{ Saturación de bases $\%$} \\
\hline Potasio (k) & 11,48 \\
\hline Calcio $(\mathrm{Ca})$ & 34,18 \\
\hline Magnesio $(\mathrm{Mg})$ & 35,65 \\
\hline Sodio $(\mathrm{Na})$ & 0,00 \\
\hline Acidez & 18,68 \\
\hline \multicolumn{2}{|c|}{ Relaciones } \\
\hline $\mathrm{Ca} / \mathrm{Mg}$ & 0,96 \\
\hline $\mathrm{Mg} / \mathrm{k}$ & 3,11 \\
\hline $\mathrm{Ca}+\mathrm{Mg} / \mathrm{k}$ & 6,08 \\
\hline $\mathrm{Ca} / \mathrm{k}$ & 2,98 \\
\hline
\end{tabular}

Las principales bases de saturación (potasio, calcio, magnesio, sodio), en los suelos del potrero con bovino, señalan un bajo nivel de calcio, altos niveles de potasio y magnesio, y un nivel aceptable sodio. En cuanto al criterio de saturación de base, nos indica que este suelo es medio, debido a que la suma de estas bases se ubica entre los valores de $50-90 \%$, y su riqueza dependerá de la capacidad de intercambio catiónica, que en este caso es baja. En base a sus relaciones presenta una carencia de calcio en la relación $\mathrm{Ca} / \mathrm{Mg}$, para $\mathrm{Ca}+\mathrm{Mg} / \mathrm{k}$, contiene un nivel adecuado para el potasio, las demás relaciones tienen niveles aceptables (Cuadro 14).

Cuadro 15. Parámetros Físico-Químico De Los Diferentes Usos De Suelos, Tesis: Evaluación de la Fertilidad de Suelos en la Granja Escuela Casiciaco Haren Alde, distrito de las Minas, provincia de Herrera, República de Panamá, 2018. 
Invest. pens. crit. (ISSN 1812-3864)

Vol. 6, No. 3, septiembre- diciembre 2018

pp. 05-21

\begin{tabular}{|c|c|c|c|c|}
\hline Usos De Suelos & $\mathrm{pH}$ & $* \mathrm{MO}(\%)$ & Textura & Color \\
\hline Cultivo De Maíz & 5,01 & 2,28 & Franco & $\begin{array}{c}\text { Pardo Amarillento } \\
\text { Claro }\end{array}$ \\
\hline Cultivo Plátano-Otoe & 4,79 & 2.81 & Franco & Pardo Amarillento \\
\hline Café & 5,67 & 2,81 & Franco & Pardo Amarillento \\
\hline Potrero con Bovino & 4,43 & 2,81 & Franco Arcilloso & Pardo \\
\hline Potrero en "descanso" & 4,54 & 1,88 & Franco & Pardo \\
\hline Bosque & 4,03 & 1,74 & Franco & Pardo Pálido \\
\hline
\end{tabular}

*Observación: MO = Materia Orgánica.

En relación al $\mathrm{pH}$, el cultivo de café presenta un $\mathrm{pH}$ ácido, debido al conjunto de árboles que interaccionan en ese uso de suelo, a diferencia de los otros usos, que presentan un $\mathrm{pH}$ muy ácido. En cuanto a la materia orgánica, en todos los usos de suelos es baja, suponiendo que son afectados por procesos de lixiviación, escorrentía superficial y sobre explotación agropecuaria. En correlación, a la textura, se observa una diferencia en potreros con bovino, interpretando que esto se debe a una acumulación de sedimentos por escorrentías superficial por años, provenientes de las otras parcelas en estudio. Respecto al color, en general todos tienen color pardo en diferentes degradaciones debido a procesos como: exceso de precipitación, lo que ha conlleva a un máximo lavado de los terrenos.

Cuadro 16. Calicatas del cultivo de café, Tesis: Evaluación de la Fertilidad de Suelos en la Granja Escuela Casiciaco Haren Alde, distrito de las Minas, provincia de Herrera, República de Panamá, 2018.

\begin{tabular}{|c|c|c|c|}
\hline \multirow{2}{*}{ Parámetros } & \multicolumn{3}{|c|}{ Análisis De Suelos De Perfiles } \\
\hline $\mathrm{pH}$ & A & B & C \\
\hline Textura & Ácido & Muy ácido & Muy ácido \\
\hline$*$ Areno & Bajo & $\begin{array}{c}\text { Franco } \\
\text { Arcilloso }\end{array}$ & Franco \\
\hline Fósforo & Traza & Bajo & Bajo \\
\hline Potasio & Medio & Medio & Traza \\
\hline Calcio & Bajo & Bajo & Bajo \\
\hline Magnesio & Medio & Medio & Medio \\
\hline Sodio & - & - & - \\
\hline Hierro & Bajo & Bajo & Bajo \\
\hline Manganeso & Medio & Bajo & Bajo \\
\hline Cobre & Alto & Medio & Alto \\
\hline Zinc & Alto & Alto & Medio \\
\hline
\end{tabular}


Invest. pens. crit. (ISSN 1812-3864)

Vol. 6, No. 3, septiembre- diciembre 2018

pp. $05-21$

\begin{tabular}{|c|c|c|c|c|}
\hline Aluminio & Bajo & Medio & Bajo \\
\hline Acidez & Bajo & Bajo & Bajo \\
\hline
\end{tabular}

Naturalmente, la calicata indica que el suelo tiene baja fertilidad. A pesar de ser un área de regeneración natural, sin ningún tipo de fertilización, esto nos muestra que la cantidad de nutrientes es baja, por horizonte. Se observa, algo de diferencia en el comportamiento de algunos microelementos, y suponemos, que están influenciados por la textura, que, de franco arenoso, en el perfil A, pasa a franco arcilloso, en el perfil B (Cuadro 16).

Cuadro 17. Calicatas del Potrero con bovino, Tesis: Evaluación de la Fertilidad de Suelos en la Granja Escuela Casiciaco Haren Alde, distrito de las Minas, provincia de Herrera, República de Panamá, 2018.

\begin{tabular}{|c|c|c|c|}
\hline Parámetros & \multicolumn{3}{|c|}{ Análisis De Suelos De Perfiles } \\
\hline \multirow{2}{*}{$\mathrm{pH}$} & Muy Ácido & Muy Ácido & Ácido \\
\hline Textura & Franco & Franco & Franco \\
\hline$*$ MO & Bajo & Bajo & Bajo \\
\hline Fósforo & Traza & Traza & Traza \\
\hline Potasio & Alto & Bajo & Medio \\
\hline Calcio & Bajo & Bajo & Bajo \\
\hline Magnesio & Bajo & Medio & Bajo \\
\hline Sodio & - & - & - \\
\hline Hierro & Bajo & Bajo & Bajo \\
\hline Manganeso & Alto & Bajo & Medio \\
\hline Cobre & Alto & Medio & Alto \\
\hline Zinc & Alto & Medio & Medio \\
\hline Aluminio & Bajo & Bajo & Bajo \\
\hline Acidez & Bajo & Bajo & Bajo \\
\hline
\end{tabular}

*Observación: MO = Materia Orgánica

Se observa que, naturalmente, la lixiviación de nutrientes se produce a lo largo de todos los perfiles de esta calicata. La textura se mantiene en todos los perfiles, es una textura bastante suelta, y esto, coincide, con lo que se observa en los análisis, en cuanto a la perdida de los nutrientes. 
Invest. pens. crit. (ISSN 1812-3864)

Vol. 6, No. 3, septiembre- diciembre 2018

pp. $05-21$

\section{Conclusiones}

En la investigación, se evaluaron seis usos de suelos, es decir, dos áreas de cultivo y dos de potreros, un área de café, y una de bosque.

En el cultivo de maíz, los suelos presentaron niveles bajos de magnesio y calcio, medio de potasio y trazas de fósforos, indicando baja fertilidad; en referencia a los microelementos se obtuvieron niveles bajos de aluminio, zinc y cobre, y medio de manganeso y hierro; la capacidad de intercambio catiónico efectiva presentó un nivel bajo lo que muestra un grado bajo de bases intercambiables.

La saturación de bases, en el cultivo de maíz, base indicó un pH muy ácido, y un desbalance en la relación del calcio respecto al magnesio y en un nivel adecuado para el potasio en la relación $\mathrm{Ca}+\mathrm{Mg} / \mathrm{k}$.

Los suelos del cultivo de plátano y otoe, mostraron niveles bajos de magnesio y calcio, medio de potasio y trazas de fósforos, indicando baja fertilidad. En relación a los microelementos se obtuvieron niveles bajos de aluminio y cobre, medio de zinc, hierro y un manganeso con nivel alto. La capacidad de intercambio catiónico efectiva presentó un nivel bajo lo que indica un grado bajo de bases intercambiables.

En la saturación de bases, en el cultivo de oteo y plátano, se notó una deficiencia de calcio con respecto al magnesio; en la relación $\mathrm{Ca}+\mathrm{Mg} / \mathrm{k}$ se mantiene un nivel aceptable para el potasio, las demás relaciones son adecuadas; con un $\mathrm{pH}$ muy ácido.

Los suelos del café, indicaron un nivel bajo de calcio, medio de potasio, magnesio y trazas de fosforo; En cuanto a los microelementos se mostró niveles bajo de cobre, zinc, y aluminio, medio de hierro y manganeso, señalando baja fertilidad; La capacidad de intercambio catiónico efectiva presentó un nivel bajo lo que indica un grado bajo de bases intercambiables.

En referencia a las bases de saturación (potasio, calcio, magnesio, sodio), en el cultivo de café, se presentó un nivel bajo de calcio, un nivel alto de potasio y magnesio, y un nivel aceptable de sodio. Hubo una deficiencia de calcio en la relación $\mathrm{Ca} / \mathrm{Mg}$, y en cuanto a la relación $\mathrm{Ca}+\mathrm{Mg} / \mathrm{k}$, se mostró un nivel aceptable para el potasio, las otras relaciones tienen un nivel aceptable; con un pH muy ácido.

Los suelos del bosque, presentan bajos niveles de calcio y magnesio, medio de potasio y trazas de fósforos, indicando baja fertilidad; En relación a los microelementos, muestran niveles bajos de zinc, medio de manganeso, cobre y aluminio, alto de hierro; La capacidad de intercambio catiónico efectiva presentó un nivel bajo lo que indica un grado bajo de bases intercambiables.

Los suelos del bosque, mostraron un nivel alto de potasio, bajo de calcio y un nivel aceptable de magnesio y sodio. Con un suelo muy ácido, y una deficiencia de $\mathrm{Ca}$ en la relación $\mathrm{Ca} / \mathrm{Mg}$; y otra deficiencia del magnesio en referencia al $\mathrm{Mg} / \mathrm{k}$; en la relación $\mathrm{Ca}+\mathrm{Mg} / \mathrm{k}$ hay un nivel adecuado para el potasio, las demás relaciones son aceptables.

En el suelo del potrero en "descanso" se mostró niveles bajos de calcio y magnesio, medio de potasio, trazas de fósforo, lo que nos indica una baja fertilidad en los suelos. En cuanto a los microelementos, 
Invest. pens. crit. (ISSN 1812-3864)

Vol. 6, No. 3, septiembre- diciembre 2018

pp. $05-21$

se presentaron niveles bajos de aluminio y zinc, un nivel alto de hierro, manganeso y cobre; La capacidad de intercambio catiónico efectiva presentó un nivel bajo lo que indica un grado bajo de bases intercambiables.

El suelo del potrero en "descanso" reveló un alto nivel de potasio y magnesio, nivel bajo de calcio y un nivel aceptable de sodio; con un $\mathrm{pH}$ neutro o básico; con carencia de $\mathrm{Ca}$ en la relación $\mathrm{Ca} / \mathrm{Mg}$, muestrando un nivel adecuado para el potasio en la relación $\mathrm{Ca}+\mathrm{Mg} / \mathrm{k}$, con las demás relaciones aceptables.

En los suelos de potrero con bovino, se manifiestan niveles bajo de potasio, calcio, magnesio y trazas de fósforo, lo que nos muestra una baja fertilidad en estos suelos; los microelementos, indicaron un nivel bajo de aluminio, medio de zinc y altos niveles de hierro, cobre y magnesio. La capacidad de intercambio catiónico efectiva mostró un nivel bajo lo que indica un grado bajo de bases intercambiables.

En los suelos del potrero con bovino, se señala un bajo nivel de calcio, altos niveles de potasio y magnesio y un nivel aceptable sodio, se presenta una carencia de calcio en la relación $\mathrm{Ca} / \mathrm{Mg}$, para $\mathrm{Ca}+\mathrm{Mg} / \mathrm{k}$ contiene un nivel adecuado para el potasio, las demás relaciones tienen niveles aceptables.

En relación al pH, el cultivo de café presenta un pH ácido, a diferencia de los otros usos que presentan pH muy ácido. En cuanto a la materia orgánica todos los usos de suelos son bajos. En correlación a la textura se observa una diferencia en potreros con bovino, interpretando que esto se debe a una acumulación de sedimentos por escorrentías superficial por años, provenientes de las otras parcelas en estudio. Respecto al color, en general todos tienen color pardo en diferentes degradaciones de tonos.

La calicata indica que el suelo tiene baja fertilidad, con limitaciones de algunos microelementos, por influencia de la textura, que de franco arenoso en el perfil A pasa a franco arcilloso en el perfil B. Se observa una lixiviación de nutrientes que desciende a lo largo de todos los perfiles de la calicata. Con una textura que se mantiene en todos los perfiles.

\section{Recomendaciones}

Para mejorar la fertilidad de los suelos, es importante llevar a cabo prácticas agroecológicas, como rotación de cultivos, rotación por mangas, aplicación de abonos orgánicos, cultivos asociados, y otras. Realizar otras investigaciones, relacionadas con las características químicas y físicas del suelo.

\section{Bibliografía}

Buckman H \& Brady N.1966. Naturaleza y Propiedades De Los Suelos. Unión Tipográfica Editorial Hispano Americana. España. 590p

Chapman D, H \& Pratt F, P. 1991. Métodos de Análisis para Suelos, Plantas y Aguas. Trillas, S.A de C.V.D.F, México.195p. 
Invest. pens. crit. (ISSN 1812-3864)

Vol. 6, No. 3, septiembre- diciembre 2018

pp. $05-21$

Fassbender W, Hans \& Bornemisza E. 1994. Química De Suelos con Énfasis en Suelos de América Latina. IICA. San José, Costa Rica. 419p. ISBN 92-9039-1243.

González, Comunicación Personal. Granja Escuela Casiciaco Haren Alde.24 de julio de 2018.

Herrera, República de Panamá.

Grijpma, et al. 1982. Producción Forestal. México. Editorial trillas, S. A. 134 p.

Kass D, C.L. 2007. Fertilidad De Suelos. Universidad Estatal a Distancia. San José, Costa Rica. 233p

Villarreal J, E; Ramos, I; Villalaz, J; Santo, A. (s/f).Proyecto: Zonificación Agroecológica de Suelos de Panamá. IDIAP. 